\title{
Optimal control of laser-generated acoustic waves in solids
}

\author{
Young Sik Kim \\ Department of Applied Science, Hong-ik University, Mapoku, Seoul 121, Korea \\ Mohsen Tadi and Herschel Rabitz \\ Department of Chemistry, Princeton University, Princeton, New Jersey 08544
}

Attila Askar

School of Sciences, Koc University, Bebek-Istanbul, Turkey

John B. McManus

Aerodyne Research Inc., Billerica, Massachusetts 01821-3976

(Received 3 August 1994)

\begin{abstract}
This paper is concerned with the design of optimal surface-heating patterns that result in focusing acoustic energy inside a subsurface target volume at a specified target time. The surface of the solid is heated by an incident laser beam which gives rise to shear and compressional waves propagating into the solid. The optimal heating design process aims to achieve the desired energy focusing at the target with minimal laser power densities and minimal system disturbance away from the target. Due to the slow motion of the thermal conduction process relative to the propagation of acoustic waves, a general formulation is derived which is the limiting case for a heat-absorbing nonconducting solid. This is consistent with the laser heating of the surface where, for the time duration of interest, thermal effects are confined to a thin layer at the surface. This simplification allows for the solution of the problem through the use of the system Green's function for the purely elastic medium where thermal expansion enters as an external force. The problem is then posed as an optimal control problem, the solution of which is the required heating pattern at the surface. The optimality conditions are secured via the conjugate gradient method and the mechanics of the elastic medium is treated by the finite element method along with using the half-space Green's function matrix. Good quality energy focusing is achieved, with the optimal designs reflecting the high directivity of the photothermally generated shear-wave patterns.
\end{abstract}

\section{INTRODUCTION}

Acoustic waves have long been recognized as a valuable tool for studying and potentially manipulating the structure, dynamics, and properties of solid materials. ${ }^{1}$ Some applications of solid acoustics require the generation of a subsurface acoustic focus. One general approach to the problem of interior focusing of acoustic energy is to act on the surface with radiant energy rather than to use transducer arrays, ${ }^{2}$ or acoustic lenses with coupling media. ${ }^{3}$ The technique of acoustic-wave generation by laser-surface interaction ${ }^{4}$ is especially interesting, since laser beams can be controlled both spatially and temporally, and energy can be delivered in a noncontacting manner in the ambient environment.

There are two common methods for generating acoustic waves in an opaque solid with a laser beam. At relatively high laser intensities, the surface is vaporized (i.e., ablated), which generates a momentum impulse normal to the surface and, hence, generates normal surface loads. A sacrificial coating can be used to allow this process to occur at laser intensities below what would be needed for bare-metal ablation. ${ }^{5}$ At laser intensities below the ablation threshold, acoustic waves are generated via heating and thermal expansion of the surface, i.e., by thermoelas- tic processes. Thermoelastic laser acoustics has been widely applied to problems of nondestructive testing and probing of materials. ${ }^{6}$ Several groups have experimented with designing laser illumination patterns for focused or directed acoustic waves. ${ }^{7-11}$

We have previously studied the application of optimal control theory to the design of normal surface force patterns for efficient generation of focused acoustic waves in solids. ${ }^{12}$ The design of those normal load patterns corresponded approximately to laser-ablation processes. The loads that resulted from that study were in the form of two collapsing rings, which contributed both shear and compressional waves to the focus. The ring surface velocities were related to the shear and compressional wave speeds, and the widths of the rings corresponded approximately to the focal target size. The acoustic isotropy of the material and the simple focusing goal of maximum energy in the target volume led to the cylindrically symmetric loads.

The purpose of the present work is to apply optimal control theory to the design of focused acoustic waves by thermoelastic processes. Thermoelastic loads are primarily tangential to the surface and produce a mixture of shear and compressional waves that is distinct from normal load wave patterns in strength and directivity. We 
will essentially repeat the steps of the earlier investigation, ${ }^{12}$ but with thermal expansion as the driving force. We also will use a geometry similar to the earlier work, a semi-infinite metal with a target volume at a depth of $\sim 0.4 \mathrm{~cm}$.

The design of the optimum surface forcing to generate a specified acoustic-wave pattern is a complicated problem, especially if one takes into account practical constraints. Optimal control theory ${ }^{13}$ can naturally take into account the physical response, as well as practical constraints in calculating the optimum application of forces, which drive a physical system to a desired state. Optimal control theory has been applied to a wide variety of problems in engineering science, especially for dynamical systems with many degrees of freedom and important constraints on the forces available to drive the system. Recently, optimal control theory has been used to calculate the optimum temporal structure of laser pulses for manipulating quantum-mechanical waves. ${ }^{14}$ Similar concepts apply to the optimal control of classical waves. In the work presented here, we apply optimal control theory to design surface heating patterns that result in the desired focused acoustic-wave structure within the solid.

In Sec. II we formulate the appropriate optimal control problem and discuss various cost functionals that incorporate practical limitations and physical objectives. In Sec. III we present and discuss numerical results.

\section{THE OPTIMAL ACOUSTIC CONTROL PROBLEM}

In this section we will describe the mathematics of the optimal control problem for generating an efficient subsurface acoustic focus driven by laser-surface heating. There are several essential elements in any optimal control problem. One derives a functional that represents the desired end state of the system (e.g., focused acoustic energy), and costs to be avoided (e.g., disturbances away from the target). The functional also contains constraints that represent the underlying physical behavior of the system (e.g., acoustic-wave equation). Minimizing the functional results in the designed system inputs (spacetime force patterns) that best satisfy the goals with the minimum costs. While there are many possible desired end states, we will use the example of maximizing acoustic energy (strain plus kinetic) within a subsurface target volume. The cost functions will represent a combination of minimal acoustic disturbance outside the target, and minimal surface driving strength. These are the same goals and costs that we employed in our earlier work. ${ }^{12}$ The end result will be an optimal laser illumination pattern.

The physical situation considered in this paper is a homogeneous, isotropic elastic solid (e.g., a metal) that occupies the lower half-space, illuminated from above by a laser beam. Part of the incident optical energy is converted to heat, which results in thermal stresses just inside the surface. The transient thermal stresses produce acoustic waves that propagate into the solid. The essential physics then is contained in the acoustic-wave equation, and the surface-heating behavior. The equation of motion for the propagation of acoustic waves is given by $\mu u_{i, j j}+(\lambda+\mu) u_{j, j i}-\gamma T_{, i}=\rho \ddot{u}_{i}, \quad(\mathbf{x}, t) \in V \times t^{+}$,

where $\lambda$ and $\mu$ are the isothermal Lame's constants, $\rho$ is the density, $\gamma=(3 \lambda+2 \mu) \alpha, \alpha$ is the linear coefficient of thermal expansion, and $T$ is the temperature rise. $V$ denotes the lower half space and $t^{+}$denotes times after the start of the laser pulse. The term $\gamma T_{, i}$ is the body force due to thermal expansion. The initial conditions and the boundary conditions are given by

$$
\begin{aligned}
& u_{i}(\mathbf{x}, 0)=\dot{u}_{i}(\mathbf{x}, 0)=0, \quad \mathrm{x} \in V, \\
& \lambda u_{j, j} n_{i}+\mu\left(u_{i, j}+u_{j, i}\right) n_{j}=\gamma T n_{i}, \quad(\mathrm{x}, t) \in S \times t^{+},
\end{aligned}
$$

where $n_{i}$ is the direction cosines of the unit outward vector normal to the surface. The heat conduction is governed by

$$
-K T_{, j j}+\rho C_{v} \dot{T}=h, \quad(\mathrm{x}, t) \in V \times t^{+},
$$

where $K$ is the thermal conductivity, $C_{v}$ is the specific heat at constant volume, and $h$ is the heat source produced by the laser beam. The appropriate boundary and initial conditions are given by

$T(\mathrm{x}, 0)=0, \quad \mathrm{x} \in V, K T_{, j} n_{j}=0, \quad(\mathrm{x}, t) \in S \times t^{+}$.

Throughout the text the standard index notation is adopted, where a summation is assumed over repeated indices, a comma followed by an index denotes spatial differentiation, and overdots indicate differentiation with respect to time.

The derivation of a complete solution to the temperature field that results from illumination of a surface with a laser having an arbitrary spatial-temporal intensity pattern is a complex problem. ${ }^{15}$ Fortunately, for our purpose we can make several assumptions that greatly simplify the description of laser surface heating. A complete treatment of heat in the solid would contain the coupling of the generated acoustic displacements back to heat. Since the acoustic loss will be small in the situations under consideration, we ignore this back coupling and have only one way coupling from heating to stress.

The spatiotemporal solution to the temperature field also can be simplified using the fact that the thickness of the heated surface layer is much smaller than the other length scales in the problem. For the case of a metal surface illuminated by a laser beam, the beam will penetrate only a small distance, the optical skin depth, which is typically of the order of $10^{-6} \mathrm{~cm} .^{16}$ During the laser pulse, the heat will diffuse to a depth given approximately by $d \approx \sqrt{\tau D}$, where $\tau$ is the pulse length and $D$ is the thermal diffusivity. The effective pulse length can be estimated by considering the form of the expected optimal heating solutions, i.e., collapsing rings with surface velocities close to the sound speeds and widths close to the target width. ${ }^{12}$ For an aluminum sample with a target radius of $0.08 \mathrm{~cm}$ at a depth of $0.32 \mathrm{~cm}$, the dwell time of the rings in any one spot will be in the range of 30-300 ns. The total time for a ring to collapse is on the order of $1 \mu \mathrm{s}$. Using a diffusivity of $0.98 \mathrm{~cm}^{2} / \mathrm{s}$ for aluminum, the heated depth will be only $\sim 1 \mu \mathrm{m}$ after $1 \mu \mathrm{s}$. The acoustic wavelengths, on the order of $0.1 \mathrm{~cm}$, are much larger than the heating depths. As long as the material behaves 
linearly, the exact distribution within the thin heated surface layer will not affect the form of the acoustic waves far from the surface. Therefore, we avoid finding explicit solutions of the thermal diffusion equation and model the surface heating in a computationally convenient form. We assume that the thermal conductivity of the material is zero $(K=0)$. We also select for the heating function an exponential with an empirical depth, $\Delta$ in the $z$ direction,

$$
h(\mathbf{x}, t)=\frac{I(r, t)}{\Delta} e^{-z / \Delta},
$$

where $h(\mathbf{x}, t)$ is the deposited heat per unit volume, $I(r, t)$ is the (absorbed) laser illumination as a function of time and radius on the surface, and $\Delta$ is the effective penetration depth. By selecting this form we also ignore lateral diffusion and closely tie together the illumination pattern, the heat source, and the temperature rise. The temperature field can then be expressed as

$$
\dot{T}(r, z, t)=\frac{h(r, z, t)}{\rho C_{v}} .
$$

Further analytical simplification is achieved by assuming a vanishingly small penetration depth $(\Delta \rightarrow 0)$, so that the temperature rise is expressed as a $\Delta$ function

$$
h(\mathbf{x}, t)=I(r, t) \delta(z) .
$$

The half-space dyadic Green's function $G_{i j}^{(+)}$for the elastic continuum ${ }^{12,17}$ can be used to describe the displacement of the solid $u_{i}(\mathbf{x}, t)$ at any point in the half space, using the thermal expansion $\left(\gamma T_{, j}\right)$ in Eq. (1) as a body force, and the temperature effects on the surface $\left(\gamma T n_{i}\right)$ in Eq. (3) as a surface traction

$$
\begin{aligned}
u_{i}(\mathbf{x}, t)= & -\int_{0}^{t} d t_{0} \int_{V} d V G_{i j}^{(+)}(\gamma T)_{, j} \\
& +\int_{0}^{t} d t_{0} \int_{S} d S G_{i j}^{(+)} \gamma T n_{j} .
\end{aligned}
$$

The thermal expansion enters Eq. (9) as a dipole force, since the Green's function in this instance is the gradient of the Green's function for a point load. ${ }^{12,17}$ After integrating by parts and using the divergence theorem, the displacement at any point in the half space is given by

$$
u_{i}(\mathrm{x}, t)=\int_{0}^{t} d t_{0} \int_{V} d V \gamma T G_{i j, j}^{(+)} .
$$

To write the displacement in terms of the applied laser intensity one needs to integrate the above relation by parts again to yield

$$
u_{i}(\mathbf{x}, t)=\int_{0}^{t} d t_{0} \int_{V} d V \gamma \dot{T} G_{i j, j}^{(H(+)},
$$

which, after substituting Eq. (7), simplifies to

$$
u_{i}(x, t)=\frac{\gamma}{\rho C_{v}} \int_{0}^{t} d t_{0} \int_{V} d V G_{i j, j}^{H(+)} h,
$$

where, $G_{i j, j}^{H(+)}$ is now the Green's function for a dipole force corresponding to a Heaviside step-function variation $H(t)$ instead of a $\Delta$ function. ${ }^{17}$ In the limit of zero penetration depth the laser intensity is related to the generated heat at the surface through Eq. (8), which, after substitution, makes it possible to integrate the above rela- tion in the $z$ direction to obtain

$$
u_{i}(x, t)=\frac{\gamma}{\rho C_{v}} \int_{0}^{t} d t_{0} \int_{S} d s G_{i j, j}^{H(+)} I(r, t) .
$$

Now that the basic physical response of the system has been described, we can construct the optimal control objective functional. We construct this functional to correspond to the goal of focusing a specified amount of acoustic energy into the target volume, at the target time. At the same time we minimize undesired system responses. We write the functional $J$ as

$$
J\left[u_{i}, h\right]=\Phi\left(t_{f}\right)+L_{1}+L_{2}+L_{3},
$$

where the individual terms are given by

$$
\begin{aligned}
& \Phi\left(t_{f}\right)=\left|\int_{V_{c}} d V \varepsilon\left(\mathbf{x}, t_{f}\right)-E_{p}\right| \\
& L_{1}=w_{1}\left(\int_{V} d V \int_{0}^{t_{f}} d t \varepsilon(\mathbf{x}, t)-w_{1}^{\prime} \int_{V_{c}} d V \varepsilon\left(\mathbf{x}, t_{f}\right)\right] \\
& L_{2}=\frac{w_{2}}{2} \int_{V} d V \int_{0}^{t_{f}} d t h^{2} \\
& L_{3}=w_{3} \int_{V} d V \int_{0}^{t_{f}} d t \int_{0}^{t} d v h
\end{aligned}
$$

with $\varepsilon(\mathbf{x}, t)$ denoting the energy density. Minimizing the function $\Phi\left(t_{f}\right)$ [Eq. (15)] corresponds to delivering a quantity of energy $E_{p}$ inside the target volume $V_{c}$ at the target time $t_{f}$. In competition with this objective are the penalty cost functions $L_{1-3}$. Minimizing $L_{1}$ [Eq. (16)] corresponds to the desire for minimizing the elastic energy of the system throughout the volume at all times except in the target volume $V_{c}$ at the target time $t=t_{f}$. The cost functionals $L_{2}$ [Eq. (17)] and $L_{3}$ [Eq. (18)] correspond to minimizing the peak heating intensities and the total energy absorbed by the surface during the process, respectively. The choice of the positive weight factors $w_{1}, w_{2}$, and $w_{3}$ is a standard practical issue in applied optimal control theory ${ }^{13}$ and allows for flexibility in balancing the role of the $\Phi$ and $L_{i}$ contributions to $J$. The numerical computations in Sec. III will illustrate some of the logic behind choosing different values for $w_{1}, w_{2}$, and $w_{3}$.

It is well known that a constrained minimization problem can be treated as an unconstrained one by introducing Lagrange multiplier functions $\psi_{i} \cdot{ }^{13}$ Hence, instead of minimizing $J$ subject to the constraint in Eq. (1), one can minimize the modified objective functional

$$
\begin{gathered}
\bar{J}\left[u_{i}, h, \psi_{i}\right]=J-\int_{V} d V \int_{0}^{t_{f}} d t \psi_{i}\left[\mu u_{i, j j}+(\lambda+\mu) u_{j, j i}\right. \\
\left.-\gamma T_{, i}-\rho \ddot{u}_{i}\right]
\end{gathered}
$$

First-order necessary conditions correspond to the vanishing of the variations of $\bar{J}$ with respect to arbitrary variations of $u_{i}, \psi_{i}$, and $h$.

The mechanical energy density, $\varepsilon(\mathbf{x}, t)$ is given as the sum of the kinetic and strain energy densities by 


$$
\varepsilon(\mathbf{x}, t)=\frac{\rho}{2} \dot{u}_{i} \dot{u}_{i}+\frac{\lambda}{2}\left(u_{k, k}\right)^{2}+\frac{\mu}{2} u_{i, j}\left(u_{i, j}+u_{j, i}\right) .
$$

The total energy density, which appears in the cost function $L_{3}$, is given by

$$
\varepsilon^{T}=\varepsilon-\gamma T u_{k, k}+\rho C_{v} T .
$$

Using the dynamical equations of motion along with the boundary and initial conditions it is easy to verify that

$$
\begin{aligned}
& \frac{d}{d t} \int_{V} d V \varepsilon(\mathbf{x}, t)=\int_{V} d V \gamma T \dot{u}_{i, i}, \\
& \frac{d}{d t} \int_{V} d V \varepsilon^{T}(\mathbf{x}, t)=\int_{V} d V \rho C_{v} \dot{T} .
\end{aligned}
$$

The above expressions are to be used in the cost functions in Eqs. (16)-(18). The expression in Eq. (23) represents the conservation of energy in the whole volume $V$. In this paper we consider the energy at the target volume in Eqs. (15) and (16) to consist of only mechanical energy, since there will not be significant transfer of elastic to thermal energy in the target volume.

The variation of $\bar{J}$ with respect to the costate function $\psi_{i}$ by construction yields the dynamical equations in Eq. (1). Similarly the equations for the costate variables (the Lagrange multiplier) are obtained through arbitrary variations of the modified cost functional $\bar{J}$ with respect to $u_{i}$, which is given by

$$
\begin{array}{r}
\mu \psi_{i, j j}+(\lambda+\mu) \psi_{j, j i}+w_{1}\left[\gamma T_{, i}-\left(t_{f}-t\right) \gamma \dot{T}_{, i}\right]=\rho \ddot{\psi}_{i}, \\
(\mathbf{x}, t) \in V \times t^{+} .
\end{array}
$$

The costate variables are required to satisfy boundary conditions on $S$ and the final conditions at $t=t_{f}$ given by

$$
\begin{aligned}
& \lambda \psi_{j, j} n_{i}+\mu\left(\psi_{i, j}+\psi_{j, i}\right) n_{j}=-w_{1}\left[\gamma T-\left(t_{f}-t\right) \gamma \dot{T}\right] n_{m}, \quad(\mathbf{x}, t) \in S \times t^{+}
\end{aligned}
$$

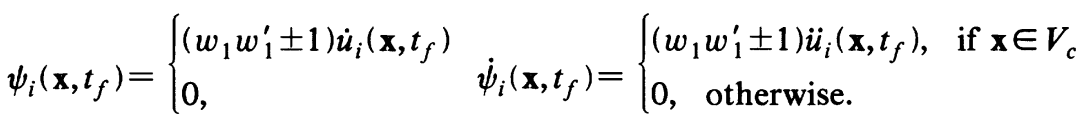

It follows that the costate function can be written in terms of the half-space dyadic Green's function as ${ }^{12}$

$$
\psi_{i}(\mathbf{x}, t)=\left(w_{1} w_{1}^{\prime} \pm 1\right) \rho \int_{V_{c}} d V\left[G_{i j}^{(-)} \ddot{u}_{j}\left(t_{f}\right)-\dot{u}_{j}\left(t_{f}\right) \dot{G}_{i j}^{(-)}\right]-w_{1} \int_{0}^{t_{f}} d v \int_{V} d V \gamma G_{i j, j}^{(-)}\left[T-\left(t_{f}-t\right) \dot{T}\right]
$$

The advanced dyadic Green's function $G_{i j}^{(-)}\left(\mathbf{x}, t ; \mathbf{x}_{0}, t_{0}\right)$ describes the $i$ th directional response at $\mathbf{x}$ corresponding to the $j$ th directional unit impulse at $x_{0}$ with time reversed. ${ }^{12}$ The two Green's functions are related through the reciprocity relation by ${ }^{18}$

$$
G_{i j}^{(-)}\left(\mathbf{x}, t ; \mathbf{x}_{0}, t_{0}\right)=G_{i j}^{(+)}\left(\mathbf{x},-t ; \mathbf{x}_{0},-t_{0}\right)=G_{i j}^{(+)}\left(\mathbf{x}, t_{0} ; \mathbf{x}_{0}, t\right) .
$$

Finally, the optimality condition is obtained through an arbitrary variation of modified objective functional over the applied heat source $\delta h$ :

$$
\left.\frac{\delta \bar{J}}{\delta h(\mathbf{x}, t)}=\frac{w_{1} \gamma}{\rho C_{v}} \iint_{t}^{t_{f}} d v u_{i, i}(v)-\left(t_{f}-t\right) u_{i, i}\right)+w_{2} h+w_{3}\left(t_{f}-t\right)-\frac{\gamma}{\rho C_{v}} \int_{t}^{t_{f}} d v \psi_{i, i}(v) .
$$

Since the heating and temperature rise are confined to a thin layer near the surface, the expressions for displacement throughout the solid may be simplified without needing to explicitly integrate the elastic equations in the $z$ direction, which reduces the computational task significantly. After taking the limit of zero penetration depth in Eq. (6), the temperature rise is a $\Delta$ function at the surface, ${ }^{17}$ and Eqs. (27) and (29) can also be integrated in the $z$ direction analytically to give

$$
\begin{aligned}
& u_{i}(\mathbf{x}, t)=\frac{\gamma}{\rho C_{v}} \int_{0}^{t} d t_{0} \int_{S} d S G_{i j, j}^{H} I, \quad(\mathbf{x}, t) \in V \times t^{+} \\
& \psi_{i}(\mathbf{x}, t)=\left(w_{1} w_{1}^{\prime} \pm 1\right) \rho \int_{V_{c}} d V\left[G_{i j}^{(-)} \ddot{u}_{j}\left(t_{f}\right)-\dot{u}_{j}\left(t_{f}\right) \dot{G}_{i j}^{(-)}\right]-\frac{w_{1} \gamma}{\rho C_{v}} \int_{t_{f}}^{t} d v \int_{S} d S G_{i j, j}^{(-) H}\left[I-\left(t_{f}-t\right) \dot{I}\right], \quad(\mathbf{x}, t) \in V \times t^{+} \\
& \frac{\delta \bar{J}}{\delta I}=\frac{\gamma}{\rho C_{v}}\left[\int_{t}^{t_{f}} d v\left\{w_{1} \dot{u}_{i, i}(v)-\psi_{i, i}\right\}-w_{1}\left(t_{f}-t\right) u_{i, i}\right]+w_{2} I+w_{3}\left(t_{f}-t\right), \quad(\mathbf{x}, t) \in V \times t^{+}
\end{aligned}
$$

Until now, the minimization of the objective functional could result in positive and negative values for the optimal heat source. However, the heat is physically a positive quantity. Therefore, we define a new control variable so that the heat is positive definite.

$$
h(\mathrm{x}, t)=\frac{1}{2} E^{2}(r, t) \delta(z),
$$

where $E$ is the square root of the absorbed laser intensity. 


\section{Laser Irradiation}

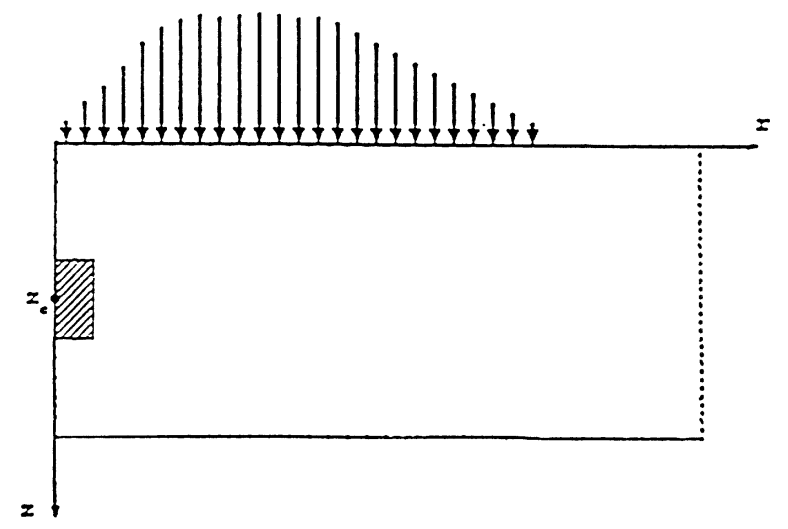

FIG. 1. Finite-element mesh for the half-space under the axial-symmetric surface laser irradiation $I(r, t)$. The shaded part denotes the target volume $V_{c}$, which is centered at $(r, z)=(0.0,0.8)$.

A new cost term for the total surface heating replaces Eq. (17) in the objective functional, Eq. (14):

$$
L_{2}=\frac{w_{2}}{2} \int_{s} d s \int_{0}^{t_{f}} d t E^{2}
$$

The optimization problem now consists of solving for the state function $u_{i}$, the costate function $\psi_{i}$, and the surface-heat source $h$ using the above three equations. Using the conjugate gradient algorithm the solution is obtained iteratively in the direction of minimizing the objective functional $\bar{J}$ until a specified convergence criterion is satisfied. More specifically, assuming an initial guess for laser intensity $I$ on the surface $S$, the displacement field $u_{i}$ in Eq. (30) can be solved for. The costate variable $\psi_{i}$ can then be solved for using Eq. (31). Finally, using both $u_{i}$ and $\psi_{i}$ a new direction to update the surface laser intensity $I$ can be found from Eq. (32). Iteration is continued until convergence is obtained.

In the next section we present computational examples that use the above algorithm. We present numerical results for the optimal surface heating patterns and discuss different cases.

\section{NUMERICAL RESULTS AND DISCUSSIONS}

Following the steps in Ref. 12, the above optimal control problem is discretized in space and time. The surface is discretized as a series of axisymmetric ring segments by the boundary element method ${ }^{19}$ and the target volume $V_{c}$ is discretized as a series of cylindrical finite element segments as shown in Fig. 1. Special care is taken in the choice of time intervals and boundary discretization in order to avoid violating the causality property of the Green's function. ${ }^{18}$ We assume the solid to be an aluminum alloy (Al-2:0:2:4) with mass density $\rho=2.77 \mathrm{~g} / \mathrm{cm}^{3}$,

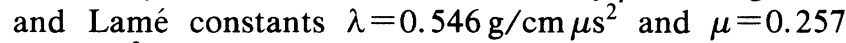
$\mathrm{g} / \mathrm{cm} \mu \mathrm{s}^{2}$. The corresponding longitudinal and shear wave velocities are, respectively, $c_{d}=0.618 \mathrm{~cm} / \mu \mathrm{s}$ and $c_{s}=0.304 \mathrm{~cm} / \mu \mathrm{s}$. The specific heat and coefficient of linear thermal expansion are given by $C_{v}=1.0 \mathrm{~J} / \mathrm{gK}$, and $\alpha=2.31 \times 10^{-5} \mathrm{~K}^{-1}$, respectively. We assume the maximum radius of the surface heating to be $R_{0} / Z_{0}=2.5$, which is divided into 25 axial-symmetric ring segments with $\Delta r=0.04 \mathrm{~cm}$. The parameter $Z_{0}$ is the dimension of the solid volume in the $z$ direction. The $z$ direction is discretized with the same mesh size as the radius, $\Delta z=0.04 \mathrm{~cm}$. Due to the simplified surface heating model discussed earlier, no integration in the $z$ direction is performed except in the target volume $V_{c}$. The target volume is a cylinder of radius $r=0.08 \mathrm{~cm}$ and height 0.16 $\mathrm{cm}$ centered at $z_{c}=0.32 \mathrm{~cm}$ from the surface. The precise target time is not important, but it must be long enough for all possible control mechanisms to act. We use a target time $t_{f}=4.5 \mu \mathrm{s}$, and a time step $\Delta t=0.03$ $\mu \mathrm{s}$.

Laser intensity

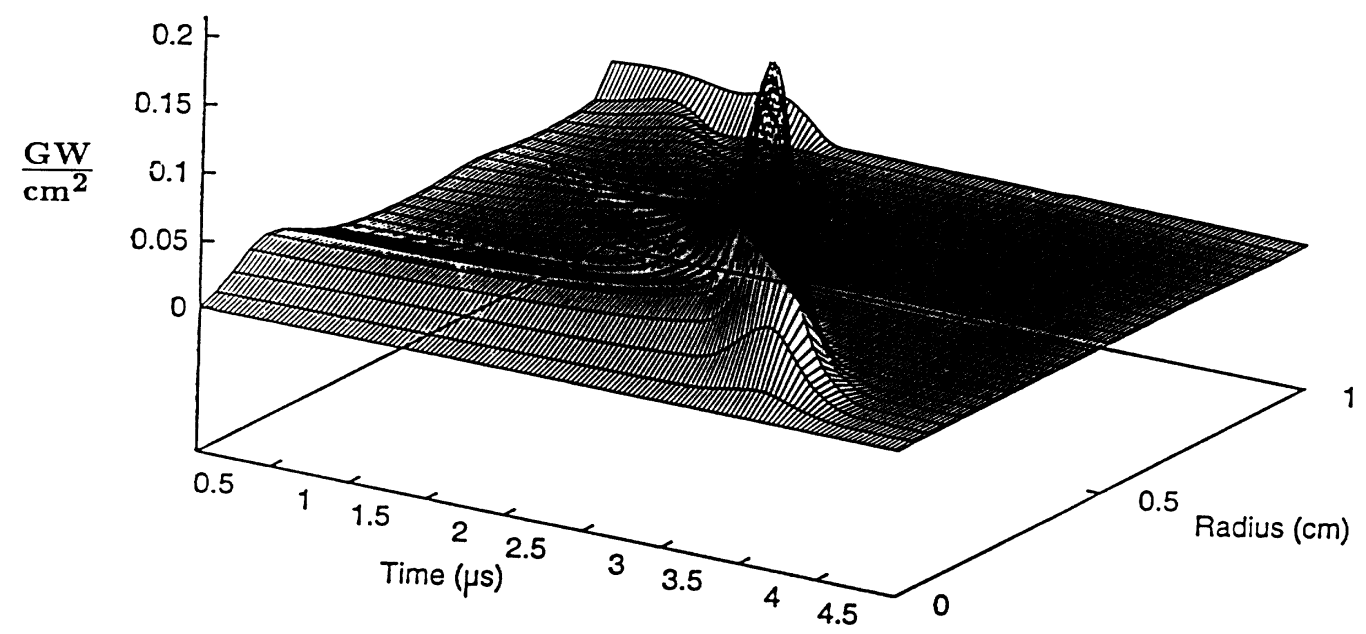

FIG. 2. Optimal laser irradiation in $\mathrm{GW} / \mathrm{cm}^{2}=10^{9} \mathrm{~W} / \mathrm{cm}^{2}$ as a function of time and space: $w_{1}=w_{2}=w_{3}=0$. 


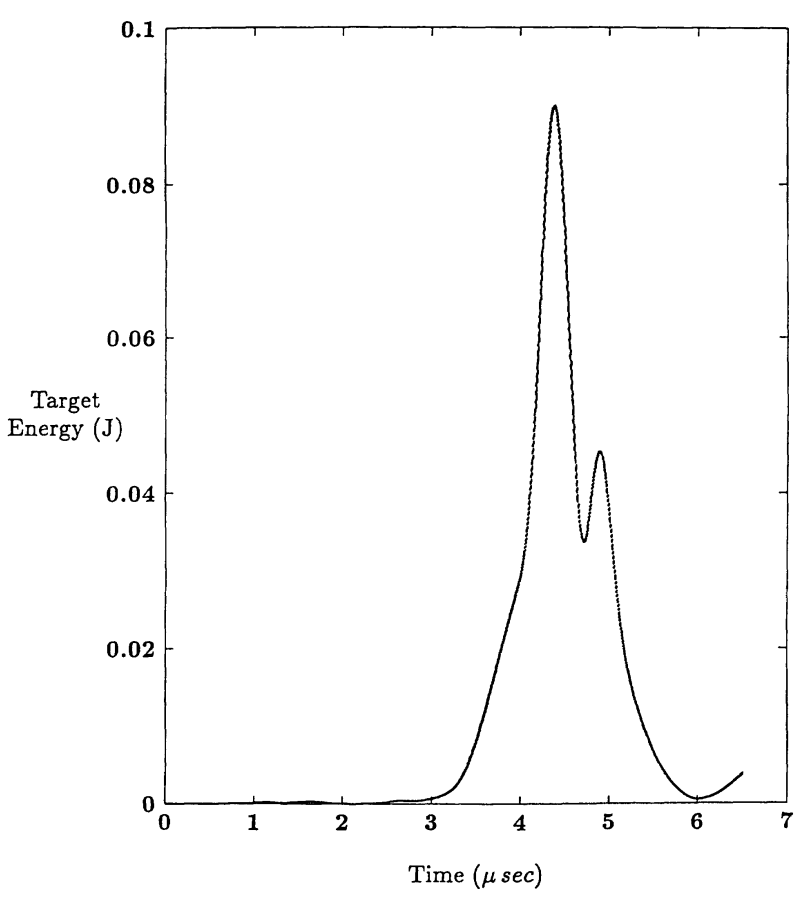

FIG. 3. Energy inside the target volume as a function of time: $w_{1}=w_{2}=w_{3}=0$.

Figure 2 shows the optimal laser irradiation of the surface when the weight factors are chosen as $w_{1}=w_{2}=w_{3}=0.0$. This corresponds to the objective of focusing $E_{p}=0.08 \mathrm{~J}$ of elastic energy inside the target volume without penalizing against either excessive heating intensities or the total energy absorbed on the surface $\left(L_{2}, L_{3}\right)$ and also without penalizing against disturbing the medium outside the target volume $\left(L_{1}\right)$. The weight factor $w_{1}^{\prime}$ is chosen to be the time-step size throughout this paper to localize the energy at the target over the smallest time scale. Figure 3 shows the computed elastic energy inside the target volume as a function of time. It can be seen in Fig. 2 that the optimal laser pulse is mostly in the shape of a ring with radius $\approx 0.2 \mathrm{~cm}$. Most of the energy is applied at time $t \approx 3.2 \mu \mathrm{s}$, which approximately $1.3 \mu$ s prior to the target time. This optimal pattern of surface heating is consistent with the fact that in the case of laser-generated surface heating most of the energy that is converted to acoustic waves is in the form of shear waves, which propagate into the solid in a highly directed manner. Figure 4 shows the directivity patterns for the shear and compressional components of the displacement inside the solid for aluminum when the surface is irradi-

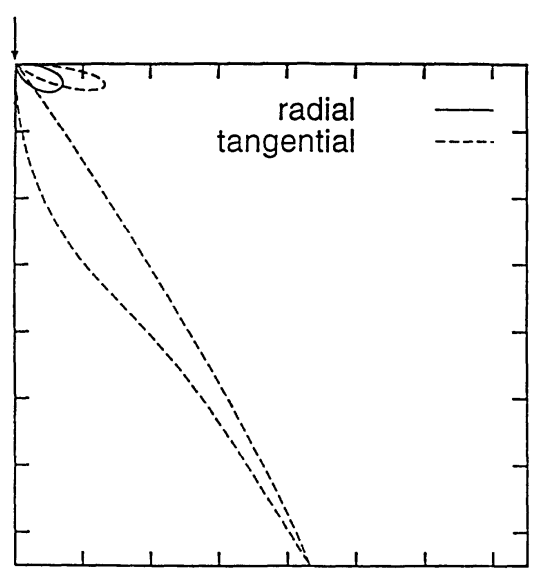

FIG. 4. Directivity pattern for radial and tangential component of the displacement inside the solid when the surface is irradiated by a point laser pulse.

ated by a pointlike laser pulse. ${ }^{17}$ This directivity pattern is a consequence of the interaction of the initial waves, and their reflection from the surface and is only a function of the Poisson's ratio $(v)$ of the material. For aluminum $(\nu=0.34)$ the peak amplitude of the shear wave is directed at an angle of approximately $32^{\circ}$ from the surface normal. The computed optimal laser illumination pattern reflects this strong angular dependence with the peak intensity at radius $\approx 0.2$ for a target depth of 0.32 $\mathrm{cm}$, corresponding to a focusing angle of $\approx 32^{\circ}$. Most of the energy is deposited $1.3 \mu$ s prior to the "target time," which is the time that it takes for the shear waves to arrive at the target. The steady ring of low amplitude laser irradiation is followed by a sharp rise in its amplitude, which makes up most of the applied heat. After $\approx 3.2$ $\mu$ s, the laser irradiation is essentially turned off, since any additional generated waves will not have sufficient time to reach the target at the specified time.

Changing the weight factors to $w_{1}=0.05, w_{2}=w_{3}=0$ corresponds to obtaining the energy focusing at the target and at the same time minimizing the disturbances elsewhere in the medium. In another case the weight factors are chosen as $w_{1}=0.05, w_{2}=0.4$, and $w_{3}=0$. This corresponds to penalizing against excessive system disturbances and against excessive heat intensity to the surface. For both of these cases the required optimal surface heating patterns exhibit similar general features as for the first case. The optimal laser irradiations correspond closely to the directivity pattern for the shear wave. A summary of the results for these cases are presented in Table I.

TABLE I. Energy yields at the target vs energy applied to the boundary.

\begin{tabular}{cccccc}
\hline \hline Cases & $w_{1}$ & $w_{2}$ & $w_{3}$ & $\begin{array}{c}\text { Total input energy } \\
\text { at the surface }(\mathrm{J})\end{array}$ & $\begin{array}{c}\text { Yield at target } \\
\text { at } t=t_{f}\end{array}$ \\
\hline 1 & 0.0 & 0.0 & 0.0 & 128 & $0.06 \%$ \\
2 & 0.05 & 0.0 & 0.0 & 50.9 & $0.15 \%$ \\
3 & 0.05 & 0.4 & 0.0 & 35.9 & $0.2 \%$ \\
4 & 0.05 & 0.0 & 0.02 & 17.6 & $0.46 \%$ \\
\hline \hline
\end{tabular}


Laser intensity

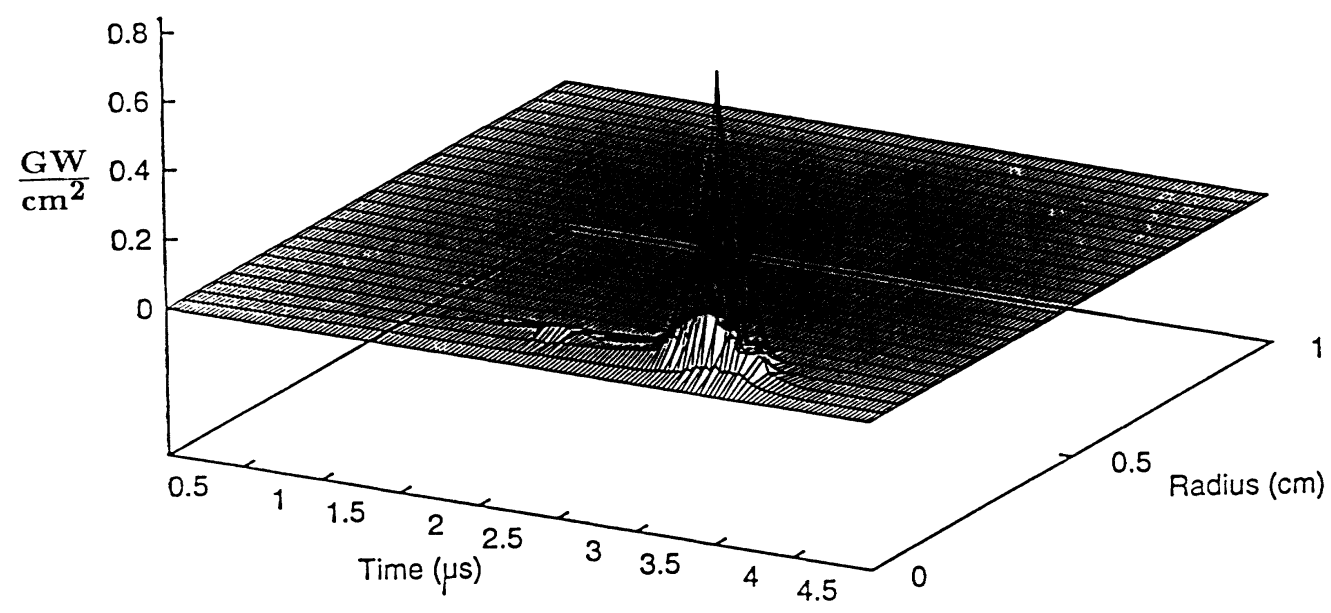

FIG. 5. Optimal laser irradiation in $\mathrm{GW} / \mathrm{cm}^{2}=10^{9} \mathrm{~W} / \mathrm{cm}^{2}$ as a function of time and space; $w_{1}=0.05, w_{2}=0$, and $w_{3}=0.2$.

Figure 5 shows the optimal laser irradiation for the case where the weight factors are chosen as $w_{1}=0.05$, $w_{2}=0$, and $w_{3}=0.02$. This corresponds to penalizing against excessive system disturbance and also against total heat applied to the surface. Figure 6 shows the corresponding time history of the energy in the target volume. For this case the initial steady heating of the surface disappears and the optimal laser irradiation is more concentrated and has a higher amplitude. For this case the objective of focusing acoustic energy is done most efficiently. Figure 7 shows the elastic energy density inside the solid at the target time as a function of space

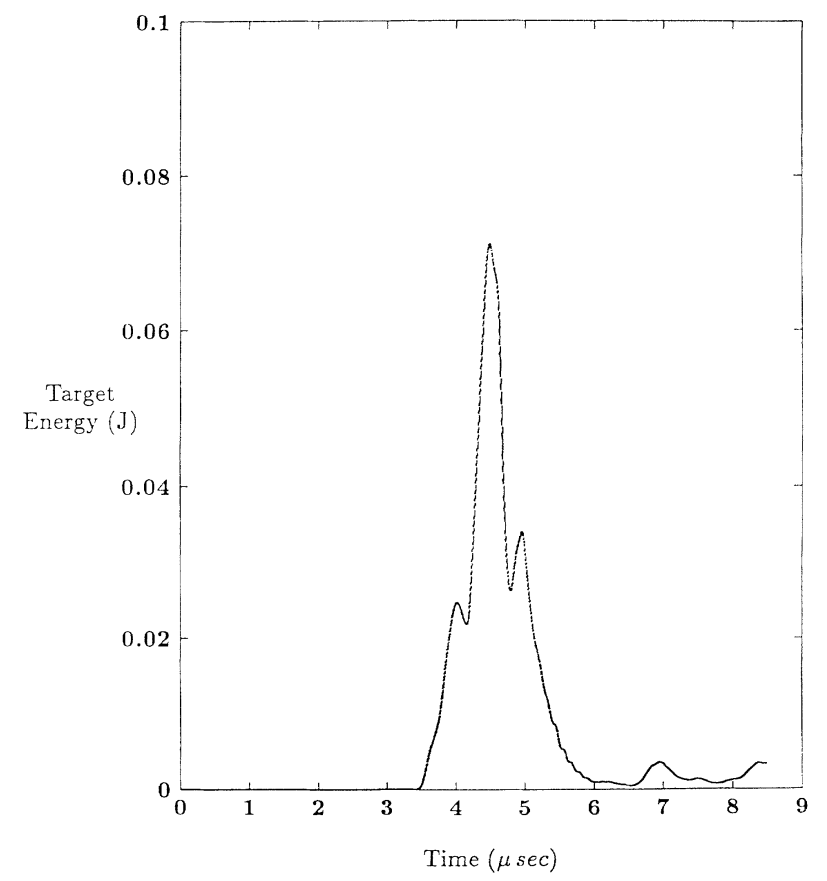

FIG. 6. Energy inside the target volume as a function of time: $w_{1}=0.05, w_{2}=0$, and $w_{3}=0.2$. coordinates. Although the required energy has reached the target, energy is also deposited in other parts of the solid. The input heat produces an integrated temperature rise, which results in persistent elastic energy inside the solid above the target volume. The leading edge of thermal expansion is what causes the required focusing of the energy at the target. Also, since there is no mechanism to remove the applied heat once the desired front is formed, a considerable amount of energy is stored in the skin of the solid in the form of the rise in temperature (not shown in Fig. 7). As a result of the strain locked in the surface, the energy inside the target volume continues to be nonzero after the initial front has passed through the target, as is shown in Fig. 6.

As expected, the optimal control algorithm gives different results for different choices of weight factors. In particular, adding the additional demand that the optimization penalizes against excessive surface heating causes the initial heating of the surface to disappear and, as a result, the acoustic focusing is obtained more efficiently. Table I shows a summary of the numerical results for the various cases. In all the cases it is possible to deposit a

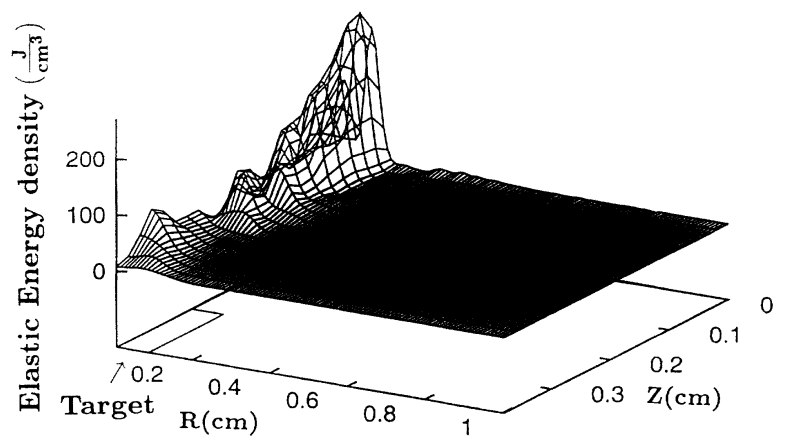

FIG. 7. Energy density $\left(\mathrm{J} / \mathrm{cm}^{3}\right)$ inside the solid as a function of space coordinates at the target time $\left(t=t_{f}\right): w_{1}=0.05$, $w_{2}=0$, and $w_{3}=0.2$. 
specified amount of energy inside the target volume. However, the efficiency in obtaining such subsurface energy focusing is considerably lower than the case where it was possible to apply normal loads to the surface. ${ }^{12,20}$ This is because most of the applied energy is stored in the solid in the form of the rise in temperature and only a small fraction of applied energy is converted to elastic energy. The calculated peak intensities are on the order of $0.2-0.8 \mathrm{GW} / \mathrm{cm}^{2}$, which is above the realistic ablation threshold. This results from the demand that $0.08 \mathrm{~J}$ of elastic energy to be focused in the target volume, which is chosen as an illustration. The target energy may be scaled down to correspond to lower intensities.

\section{CONCLUSIONS}

In this paper we studied the use of laser-generated boundary heat sources for focusing acoustic energy in a subsurface target volume. A simple formulation for the thermoelastic coupling at the surface was presented, which is the limiting case for a heat absorbing nonconducting solid. This approximation models the situation where the surface is heated by the incident laser pulse and the thermal effects are confined to a small region just inside the surface for the time duration of interest. The laser surface-heating pattern was derived as a solution to an optimal control problem. This formulation allows the designer to obtain the required energy focusing and at the same time penalize against undesired system responses.
In this paper we considered cost functionals, which correspond to penalizing against system disturbances away from the target volume and also against excessive heating intensity and the total energy absorbed on the surface during the process. The results indicate that in all the cases it is possible to obtain an optimal heating pattern on the surface that results in focusing of the acoustic energy inside the target volume. The percentages of energy yields at the target (compared to the input energy at the surface) is considerably lower than the case where it was possible to apply normal forces to the surface. ${ }^{12}$ An additional result of this work is that using laser generated surface heating to focus acoustic energy does not require sweeping of the laser pulse. The acoustic generation mechanism is predominantly controlled by the directivity patterns of the shear waves inside the medium and the optimal patterns reflect that directivity. This is different from the case where direct normal force was used to generate the desired subsurface energy focusing ${ }^{12}$ for which the optimal loading calls for sweeping normal loads in the form of rings.

\section{ACKNOWLEDGMENTS}

The authors acknowledge support for this work from the Department of Energy. Y.S.K. acknowledges support by the Korea Science and Engineering Foundation under Contract Nos. 931-0300-002-2 and by Hong-ik University.
${ }^{1}$ Y. H. Pao, ASME J. Appl. Mech. 50, 1152 (1983).

${ }^{2}$ G. S. Kino, Proc. IEEE, 67, 484 (1979).

${ }^{3}$ C. F. Quate, A. Atalar, and H. K. Wickranasighe, Proc. IEEE, 67, 1092 (1979).

${ }^{4}$ C. B. Scruby and L. E. Drain, Laser Ultrasonics-Techniques and Applications (Hilger, New York, 1990).

5J. A. Fox, Appl. Phys. Lett. 24, 461 (1974).

${ }^{6}$ A. C. Tam, Rev. Mod. Phys. 58, 381 (1986).

${ }^{7}$ A. J. A. Bruinsma and J. A. Vogel, Appl. Opt. 27, 4690 (1988).

${ }^{8}$ J. W. Wagner, A. D. W. McKie, J. B. Spicer, and J. B. Deaton, Nondestructive Evaluation 9, 263 (1990).

${ }^{9}$ Y. H. Berthelot and J. Jarzynski, Nondestructive Evaluation 9, 271 (1990).

${ }^{10}$ R. J. Von Gutfeld, D. R. Vigliotti, C. S. Ih, and W. R. Scott, Appl. Phys. Lett. 42, 1018 (1983).

${ }^{11}$ P. Cielo, F. Nodeau, and M. Lamontagne, Ultrasonics 55 (1985).
${ }^{12}$ Y. S. Kim, H. Rabitz, A. Askar, and J. B. McManus, Phys. Rev. B 44, 10 (1991).

${ }^{13}$ A. E. Bryson, Jr. and Yu-Chi Ho, Applied Optimal Control (Wiley, New York, 1975).

${ }^{14}$ S. Shi, A. Woody, and H. Rabitz, J. Chem. Phys. 88, 6870 (1988).

15J. F. Ready, J. Appl. Phys. 36, 462 (1965).

16J. D. Jackson, Classical Electrodynamics (Wiley, New York, 1975).

${ }^{17}$ L. R. F. Rose, J. Acoust. Soc. Am. 75, 723 (1984).

${ }^{18}$ P. M. Morse and F. Feshbach, Methods of Theoretical Physics (McGraw-Hill, New York, 1953).

${ }^{19}$ C. A. Brebbia, The Boundary Element Method for Engineers (Pentch, London, 1978).

${ }^{20}$ M. Tadi, H. Rabitz, A. Askar, J. H. Prevost, Y. S. Kim, and J. B. McManus (unpublished). 


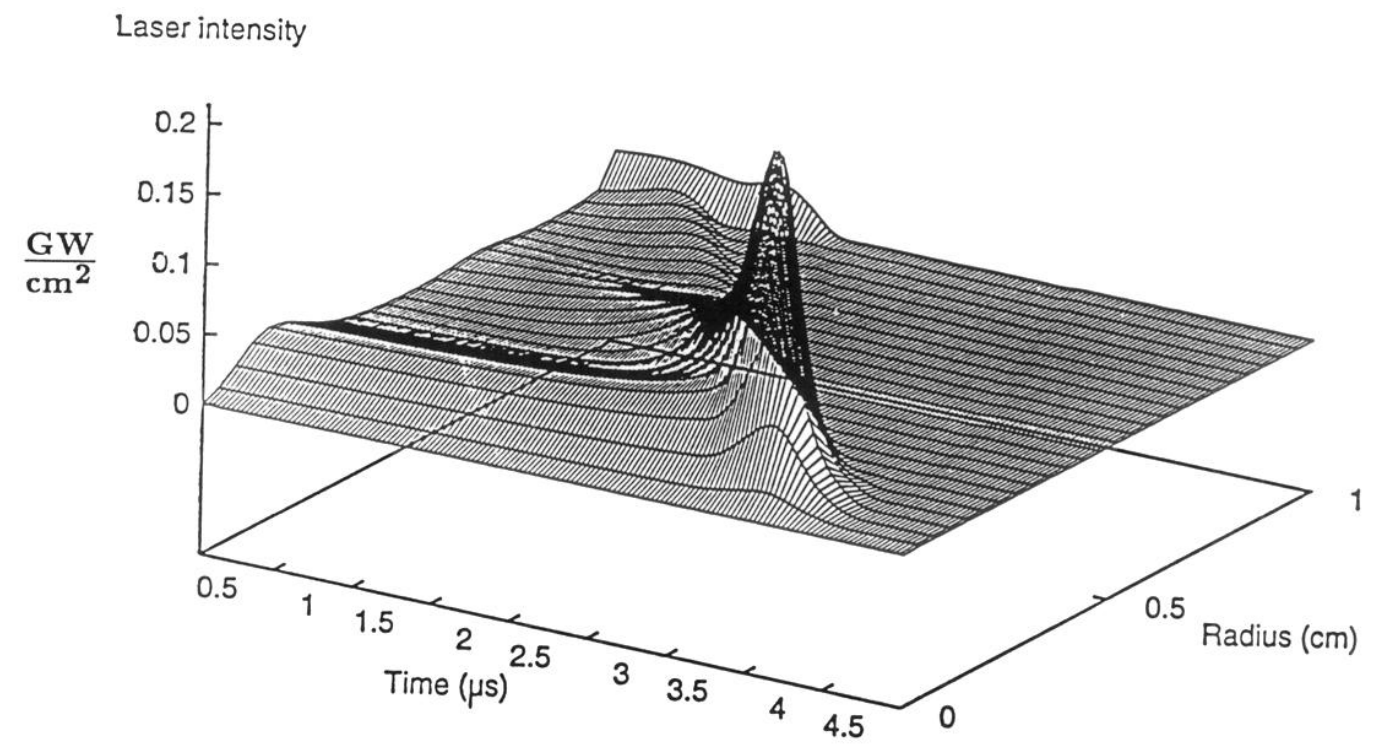

FIG. 2. Optimal laser irradiation in $\mathrm{GW} / \mathrm{cm}^{2}=10^{9} \mathrm{~W} / \mathrm{cm}^{2}$ as a function of time and space: $w_{1}=w_{2}=w_{3}=0$. 


\section{Laser intensity}

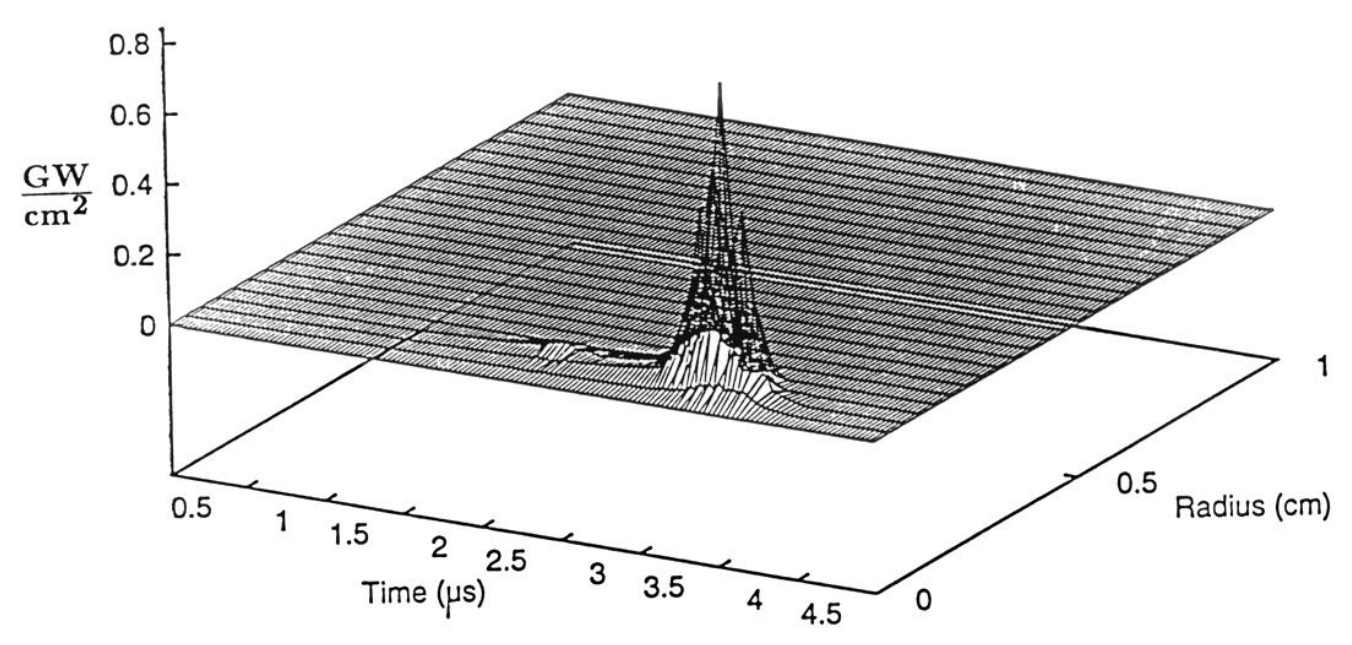

FIG. 5. Optimal laser irradiation in $\mathrm{GW} / \mathrm{cm}^{2}=10^{9} \mathrm{~W} / \mathrm{cm}^{2}$ as a function of time and space; $w_{1}=0.05, w_{2}=0$, and $w_{3}=0.2$. 


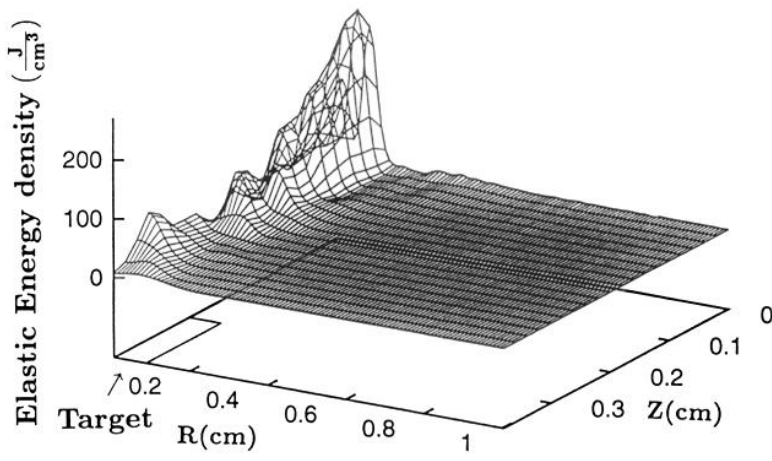

FIG. 7. Energy density $\left(\mathrm{J} / \mathrm{cm}^{3}\right)$ inside the solid as a function of space coordinates at the target time $\left(t=t_{f}\right): w_{1}=0.05$, $w_{2}=0$, and $w_{3}=0.2$. 